\title{
The accumulation of femtosecond laser radiation energy in crystals of lithium fluoride
}

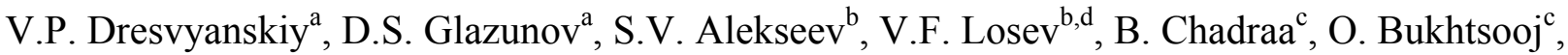 \\ N. Baasankhuu ${ }^{\mathrm{c}}$, B. Zandan ${ }^{\mathrm{c}}$, E.F. Martynovich ${ }^{\mathrm{a}}$ \\ a Irkutsk Branch of Institute of Laser Physics SB RAS, 664033, Irkutsk, 130a Lermontov str., Russia, \\ e-mail: filial@ilph.irk.ru; \\ ${ }^{b}$ Institute of High Current Electronics SB RAS, 634055, Tomsk, 2/3 Akademichesky Avenue, Russia \\ ${ }^{\mathrm{c}}$ Institute of Physics and Technology, Mongolian Academy of Sciences, Ulaanbaatar \\ 51, Mongolia \\ dNational Research Tomsk Polytechnic University, 30 Lenin Ave., 634034, Tomsk, Russia
}

\begin{abstract}
We present the results of studies of energy accumulation during the non-destructive interaction of extremely intense near infrared laser radiation with model wide band gap dielectric crystals of lithium fluoride, when the intensity of pulses is sufficient for effective highly nonlinear absorption of light and for the excitation of the electron subsystem of matter and the energy of pulses is still not sufficient for significant heating, evaporation, laser breakdown or other destruction to occur. We studied the emission of energy in the form of light sum of thermally stimulated luminescence accumulated under conditions of self-focusing and multiple filamentation of femtosecond laser radiation. It was established that it's the $\mathrm{F}_{2}$ and $\mathrm{F}_{3}{ }^{+}$color centers and supplementary to them centers of interstitial type which accumulate energy under the action of a single femtosecond laser pulses. When irradiated by series of pulses the $F_{3}, F_{3}{ }^{-}$and $F_{4}$ centers additionally appear. $F_{2}$ centers are the main centers of emission in the process of thermally stimulated luminescence of accumulated energy. The interstitial fluoride ions (I-centers) are the kinetic particles. They split off from the $\mathrm{X}_{3}{ }^{-}$centers in the result of thermal decomposition of latter on the I-centers and molecules $\mathrm{X}_{2}^{0}$. I-centers recombine with $\mathrm{F}_{3}{ }^{+}$ centers and form $\mathrm{F}_{2}$ centers in excited state. The latter produce the characteristic emission spectrum emitted in the form of thermally stimulated luminescence.
\end{abstract}

Keywords: laser, pulse, femtosecond, self-focusing, filamentation, color center, luminescence, thermoluminescence, light sum.

\section{INTRODUCTION}

Dielectric mediums are transparent in a wide spectral range from the vacuum ultraviolet to mid-IR region. Visible and near-IR radiation generated by standard femtosecond lasers in linear regime of excitation is generally not absorbed by such mediums. However, when the intensity of laser radiation is high the processes of highly nonlinear interaction of light with matter begin. Nonlinear absorption of light is ensured by the excitation of the electronic subsystem of the dielectric, mostly by electron-hole pairs and excitons [1]. The storage of energy in pure perfect dielectric, for example, in ionic crystals, in the form of intrinsic electronic excitations is possible only during the short lifetime of these excitations. Longer-term accumulation of energy on the period of seconds, days and years is provided by either structural changes in the atomic core subsystem of a solid body as a result of its interaction with the excitations of the electron subsystem, or by injection of alloying additions which form deep traps for electrons and holes [3].

It is known that luminescent defects are effectively created by intense femtosecond pulses during the irradiation of dielectric crystals, predominantly with excitonic mechanism of defect formation [1-2]. Defects induced by femtosecond laser pulses in such crystals are simple and aggregate color centers, characteristic for radiation staining [4-5].

International Conference on Atomic and Molecular Pulsed Lasers XII, edited by Victor F. Tarasenko,

Andrey M. Kabanov, Proc. of SPIE Vol. 9810, 98100N · (c) 2015 SPIE

CCC code: $0277-786 \mathrm{X} / 15 / \$ 18 \cdot$ doi: $10.1117 / 12.2225415$

Proc. of SPIE Vol. $981098100 \mathrm{~N}-1$ 
The aim of this research was to study the mechanisms of interaction between electronic and atomic core subsystems of a solid body, the energy transfer between them, the formation of structural defects accumulating energy of laser radiation, the transfer of this energy to the centers of recombination and its emission.

We have conducted studies on the model dielectric crystals with predominantly ionic bond, for the most part on the crystals of lithium fluoride (width of the forbidden zone $\sim 14 \mathrm{eV}$ ). We studied non-destructive interaction of extremely intense laser radiation (photon energy of $1.3-1.5 \mathrm{eV}$ ) with the dielectric; in such conditions the intensity of pulse is sufficient for effective highly nonlinear light absorption and excitation of the electron subsystem of the matter and the energy of the pulses is insufficient for significant heating, evaporation, laser breakdown or other destruction of the sample to occur. To provide such conditions we used the intense laser pulses of small duration.

\section{EXPERIMENT}

We have conducted studies on the model wide-gap crystals of lithium fluoride (LiF) grown with the Kyropoulos method in air atmosphere. The samples were fabricated in the form of parallelepipeds with a cross section of about $10 \times 10 \mathrm{~mm}$ and a length of 30-35 $\mathrm{mm}$. The natural fractures along the cleavage planes were the surfaces of the samples, the frontal surfaces of the crystals were polished, the laser radiation entered samples through these frontal surfaces. Initially the samples appear clear and colourless. Pre-measured IR absorption spectra indicated presence of impurities identified as oxygen and hydroxyl impurities according to the literature data.

We performed experiments on irradiation of the crystals by femtosecond laser radiation on installation which included a titanium-sapphire laser generating pulses with duration of $50 \mathrm{fs}$ with energy of about $6 \mathrm{~mJ}$ and the maximum of the emission spectrum of this laser is at a wavelength of $950 \mathrm{~nm}$. Exciting pulses were focused with a lens with a focal length of $425 \mathrm{~mm}$.

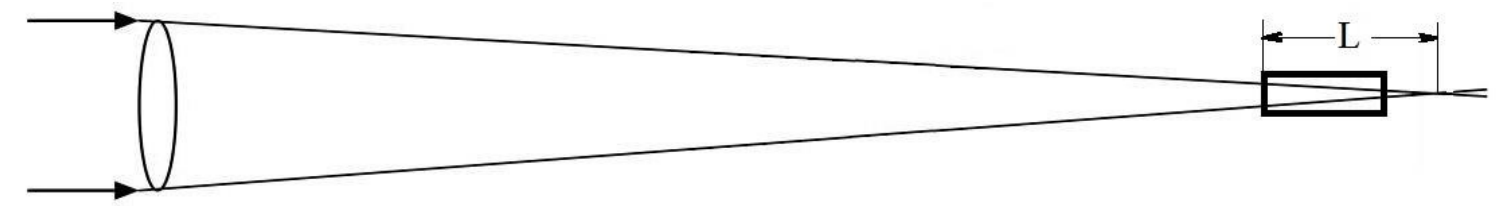

Figure 1. The scheme of experiments on irradiation of LiF crystals by femtosecond laser pulses in lowaperture external focusing mode.

The irradiation scheme is shown in Fig. 1. In the experiments we used the mode of interaction of light with the sample, corresponding to the conditions of low aperture external focusing when the exciting laser radiation pulses are focused with a lens with a long focal length. The total energy of the laser radition passed through the sample was determined by the number of pulses.

The study of the defect formation topology an photoluminescence of th samples irradiated by laser radiation was carried out on a highly sensitive scanning confocal microscope with picosecond time resolution MicroTime 200 by PicoQuant Gmbh with spatially-selective time-correlated scoring of single photons. The microscope enables to record longitudinal and transverse spatial distribution of the concentrations of the defects created by laser radiation and to imaging of microobjects within the irradiated volume of medium in luminescent radiation with scanning step of $10 \mathrm{~nm}$ and selection of images by the luminescence decay time.

The photoluminescence spectra when excited by picosecond laser pulses in the spectral region of absorption of induced color centers from 370 to $640 \mathrm{~nm}$ was recorded by a spectrometer Ocean Optics 65000.

A technique based on the phenomenon of thermally stimulated luminescence (TSL) was applied for the study of axial (in the direction of laser radiation propagation) spatial distribution of the accumulated energy. We carried out studies for the four samples irradiated by series of femtosecond pulses (10 to 1000). After irradiation each sample was cut into plates with a thickness of 1-2 $\mathrm{mm}$. A specialized assembly was used to record thermoluminescence curves and to determine the value of the stored light sum for each of the plates in the temperature range from $20{ }^{\circ} \mathrm{C}$ to $400{ }^{\circ} \mathrm{C}$ with a constant heating 
rate of $0.25 \mathrm{deg} / \mathrm{sec}$. After determining the value of the light sum stored in each of the plates we defined the dependences of the axial spatial distribution of accumulated energy in each of the four samples.

\section{RESULTS AND DISCUSSION}

The experiments showed that the crystals of lithium fluoride irradiated by intense femtosecond laser radiation accumulate energy which can be discharged in subsequent heating in the form of thermally stimulated luminescence (Figure 2). The main TSL peaks are in the region of 200-300 K. Our data for the most part match with the results of study of TSL of nominally pure radiation-colored crystals of lithium fluoride described in [6].

The presented axial dependences show that energy is not accumulated in the opening part of the crystal. The value of light sum reaches its maximum magnitude at the distance of about $7 \mathrm{~mm}$ from the surface of the samples which corresponds to the distance of self-focusing of the exciting laser radiation. The dependencies are "ragged", nonmonotonic in nature after the influence of the single femtosecond pulses of laser radiation or the small series of them. When one increases the number of pulses the dependences of the stored light sum become smooth and represent an asymmetric curve with one maximum (Fig. 2 b). To clarify the reasons of such behavior of these dependences we conducted microscopic examination of the topography of the channels within which laser pulses proceeded in crystal. Since luminescent color centers were created in crystal under the action of these pulses the research has been performed with use of fluorescent microscopy with time resolution.

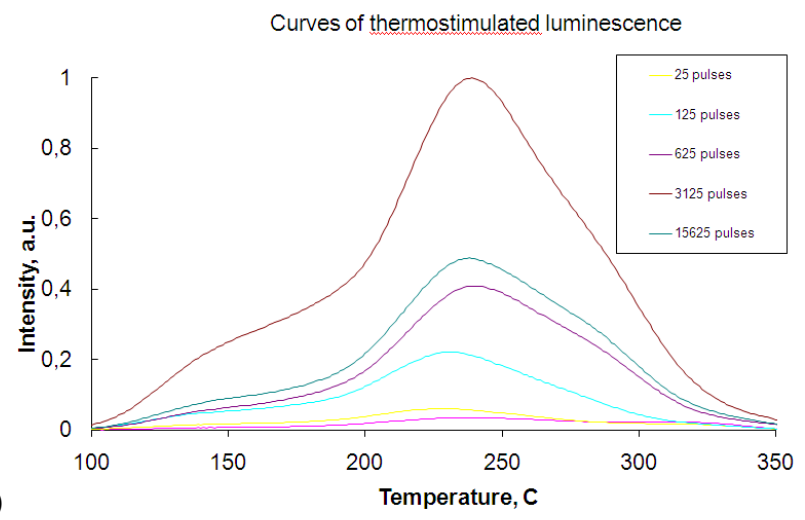

a)

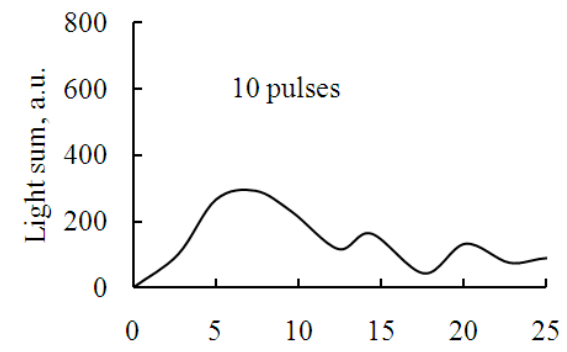

b)

Length of the crystal, $\mathrm{mm}$

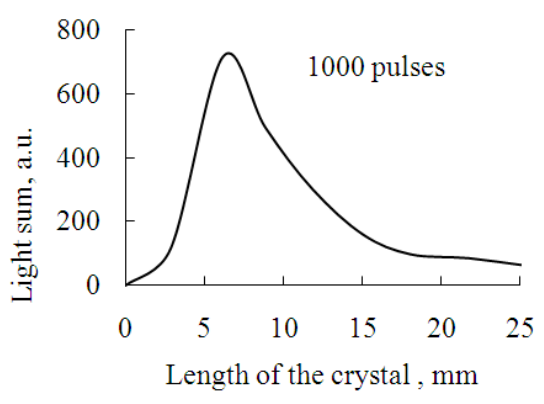

Figure 2. Curves of thermostimulated luminescence (a) and axial spatial distribution of the TSL light sum along the length of the crystal (b) of the LiF crystal samples irradiated with different number of femtosecond laser radiation pulses.

Photos of the luminescent channels after irradiation of the samples by femtosecond pulses of titanium-sapphire laser obtained with use of a microscope Olimpus IX 71 are presented in Figure 3. Spurs (traces of filaments) and the channels are visualized by excitation of photoluminescence of products of photochemical reactions which occurred in crystals during and after the irradiation by femtosecond pulses. The wavelength of radiation exciting luminescence is $450 \mathrm{~nm}$. A more detailed study of the topography of the defect formation and photoluminescence of the samples irradiated by laser 
radiation has been carried out with use of the highly sensitive scanning confocal microscope with picosecond time resolution MicroTime 200 by PicoQuant Gmbh with spatially-selective time-correlated scoring of single photons. The results of scanning of longitudinal and transverse dimensions of a single spur formed by a single filament of a femtosecond pulse at near-threshold (for filamentation) intensity are presented in Figure 4a.
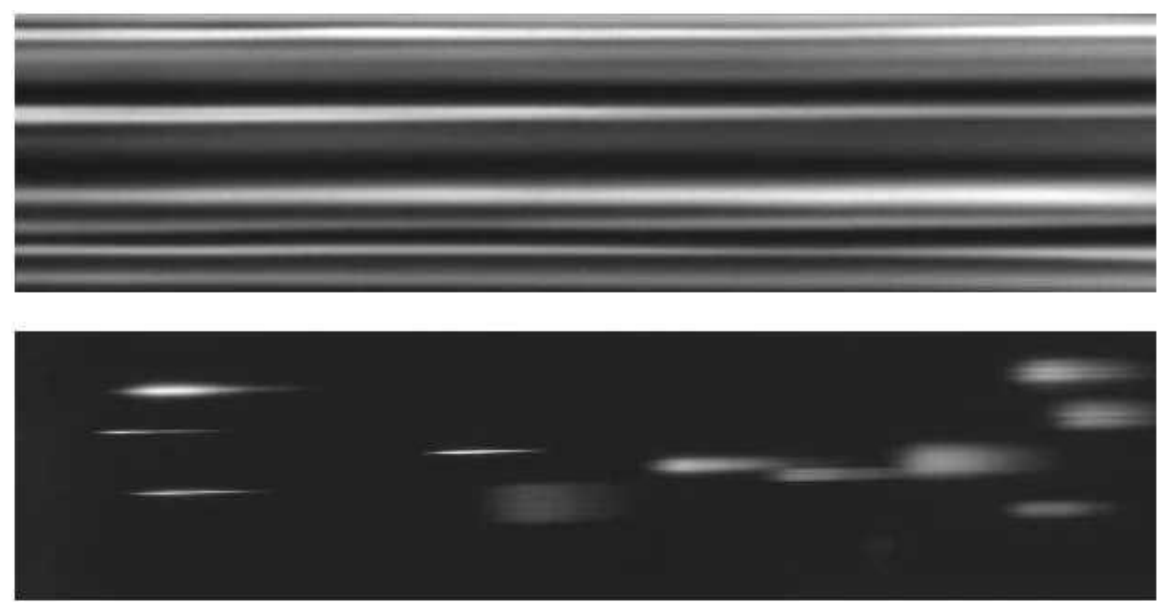

Figure 3. Photos of the spatial distribution of the intensity of photoluminescence of the centers formed in crystals by irradiation of periodic sequence of pulses (top) and by single femtosecond pulses (bottom). Size is $80 x 300 \mu \mathrm{m}$.

a)

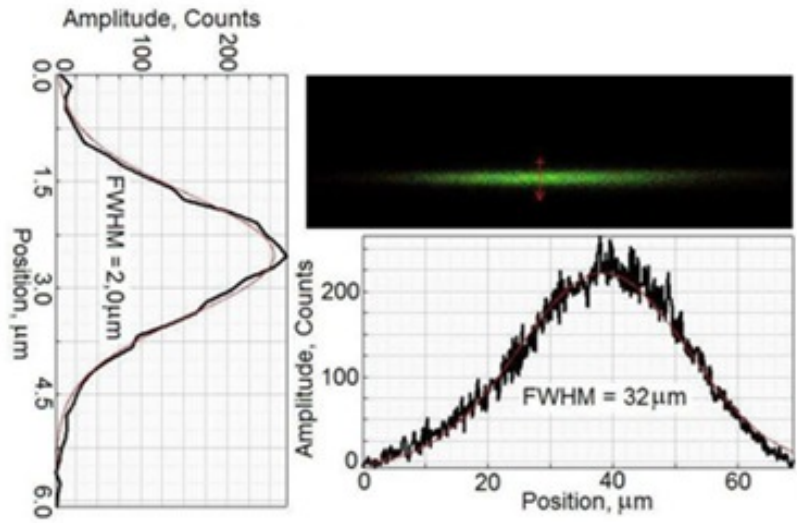

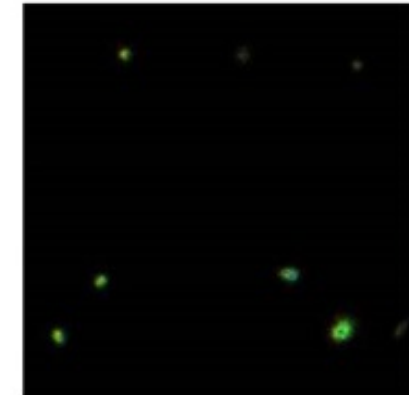

b)

$$
25 \text { pulses }
$$

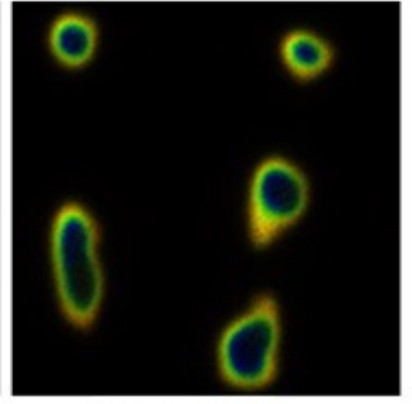

1000 pulses

Figure 4. The results of scanning of longitudinal and transverse dimensions of a single spur (a) and transverse distribution of intensity of luminescence of color centers in the spurs (the scan area is $25 \times 25 \mu \mathrm{m}$ ) formed by laser radiation (b). The minimum length of the spur is equal to $32 \mu \mathrm{m}$, its minimum diameter is $2.0 \mu \mathrm{m}$. 
The presented results of microscopic examination show that the "ragged" nonmonotonic nature of the axial dependence of the stored light sum (Fig. 1a) is determined by the fact that the storage takes place in the areas of self-focusing filaments of laser radiation which are small in size (their length is $\sim 30 \mathrm{~mm}$, their diameter is $\sim 2 \mu \mathrm{m}$ ) in the initial stages of irradiation and their number is small. When number of laser pulses increases the length and diameter of the spurs induced in the crystal by filaments also increase and the axial dependence of the accumulated energy is smoothed (Fig. 1b). The lack of accumulated energy in the opening part of the crystal in Fig. 1 is due to the fact that the energy storage occurs only in conditions of filamentation in consequence of self-focusing. In turn self-focusing occurs at a certain distance corresponding to the self-focusing distance, so there is a spatial delay in the storage of energy in crystal.

The results of the research of spectral-kinetic characteristics of the defects induced by the series of 10 and 1000 femtosecond laser pulses are presented in Fig. 4 and 5 respectively. Luminescence was excited by laser with radiation wavelength $375,470,532$ and $640 \mathrm{~nm}$. The luminescence was detected through interference filters with a $\Gamma$-shaped response. The dip in the spectra at a wavelength of $\sim 945 \mathrm{~nm}$ is due to the absorption spectral line of the fiber optic cable used to transport the luminescence to the spectrometer.
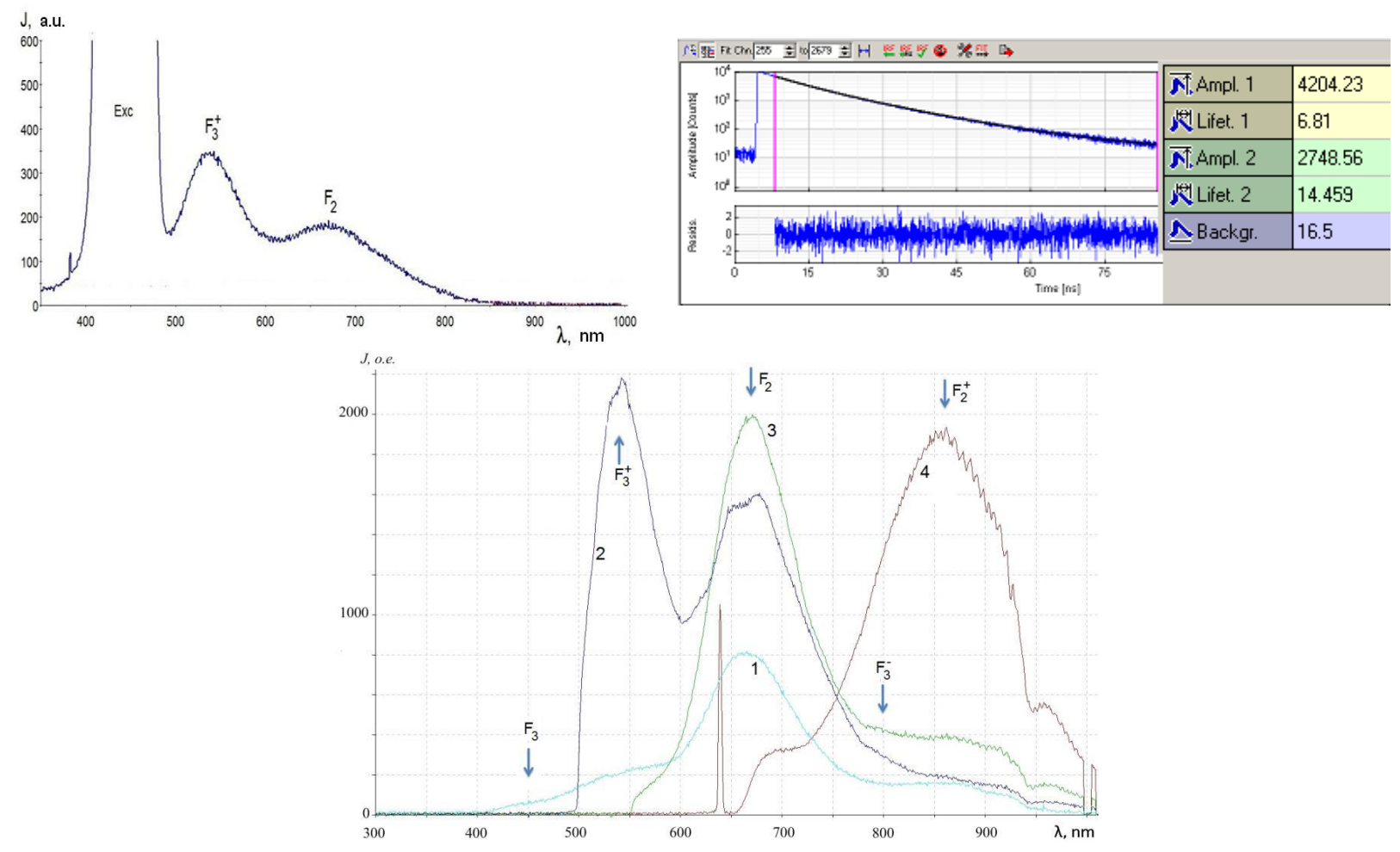

Figure 5. Spectra and kinetics of the photoluminescence of color centers of the crystal irradiated by a) series of 10 femtosecond pulses; b) series of 1000 femtosecond pulses.

From the results presented in Fig. 5 one can see that in the luminescence spectrum there are luminescence bands of $F_{2}$ color centers with the radiation wavelength maximum $\lambda=680 \mathrm{~nm}$ and $\mathrm{F}_{3}{ }^{+}$-centers with maximum at $\lambda=540 \mathrm{~nm}$, characteristic of radiation staining of lithium fluoride crystals. The measured values of the luminescence decay time constants are 14.5 and $6.8 \mathrm{~ns}$ in lithium fluoride samples irradiated by laser radiation and these values are also close to the characteristic values of the decay times of $\mathrm{F}_{2}$ and $\mathrm{F}_{3}{ }^{+}$-centers in radiation-colored crystals, which are $16 \mathrm{~ns}$ and $8 \mathrm{~ns}$ respectively, and both sets of values coincide within the measurement error. With increasing radiation exposure more complex aggregate color centers $\mathrm{F}_{3}, \mathrm{~F}_{3}{ }^{-}$and $\mathrm{F}_{4}$ shown in Figure $5 \mathrm{~b}$ appear. 
With increasing number of laser irradiation pulses the magnitude of the light sum of thermally stimulated luminescence emitted by irradiated crystals grows superlinear in the beginning then reaches a maximum and then decreases (Fig. 6a). The discovered effect of the accumulated light sum saturation is due to the fact that the concentration, aggregation degree and nomenclature of color centers are higher in the central paraxial part of the spurs than in the peripheral part, as shown by the results of micro-spectral-temporal luminescence sensing of the sections of spurs (Fig. 4b) induced by laser radiation. Therefore the luminescence yield of $\mathrm{F}_{3}{ }^{+}$and $\mathrm{F}_{2^{-}}$centers, which play an important role in the thermoluminescence process in the periphery, is higher in periphery than in paraxial region. Moreover the diameters of the spurs and consequently the volumes of their paraxial areas with low luminescence yield increase along with the growth of number of irradiation pulses, which causes weakening of the thermally stimulated luminescence intensity.
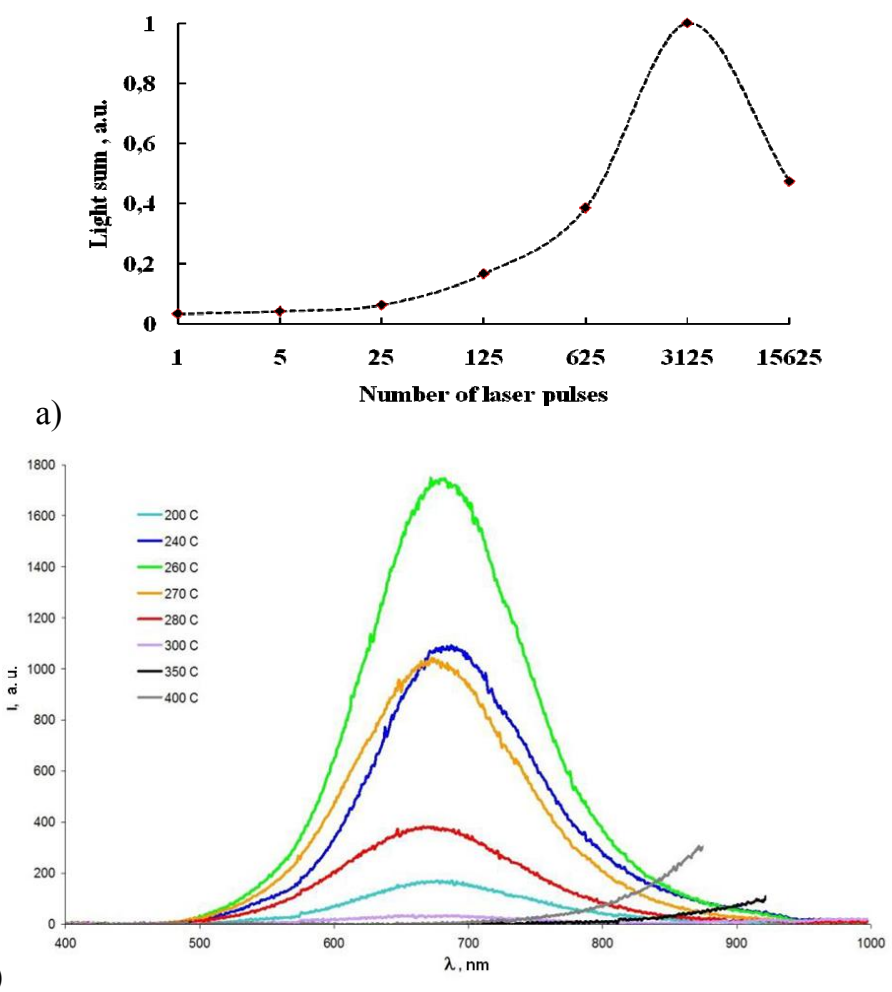

Figure 6. The dependence of TSL light sum emitted by the crystals on the number of laser irradiation pulses (a) and spectra of thermally stimulated luminescence of $\mathrm{LiF}$ crystals at different temperatures measured in the process of thermoluminescence (b).

The spectral scanning of TSL peaks shows that $\mathrm{F}_{2}$ centers make the main contribution to the luminescence. It is evident from the spectra shown in Figure 6b. It is the main emission center emitting quanta of luminescence at the final stage of thermoluminescence. The question arises about the nature of kinetic particles and centers of their recombination, which are able to form excited $F_{2}$ centers. It is important to understand what particles are kinetic, i.e. which particle is released from the place of localization and recombine with other particles which leads to the emission of TSL quanta, in our case - to the emission of the photoluminescence quanta of $F_{2}$ centers. One can assume that interstitial fluoride ions (I-centers) are the kinetic particles. They split off the $\mathrm{X}_{3}{ }^{-}$centers in result of thermal decomposition of latter into the I-centers and molecules $\mathrm{X}_{2}^{0}$. I-centers recombine with $\mathrm{F}_{3}{ }^{+}$centers turning them into $\mathrm{F}_{2}$ centers in excited state, i.e., turning them into the excited centers of emission which provide the characteristic emission spectrum of $F_{2}$ centers emitted in the form of thermally stimulated luminescence. 


\section{CONCLUSION}

Presented results unambiguously indicate the color center typical of radiation-colored wide-gap crystals of lithium fluoride are effectively created in the crystals of this type under influence of near infrared femtosecond laser radiation. That is to say the color centers are the main type of defects that accumulate energy in the form of stored light sum in the interaction of femtosecond laser pulses with wide-gap crystals.

It is known that the first stage of the color centers formation is the creation of electron-hole pairs and the energy required to create them is greater than the width of the forbidden zone, which is about $13-14 \mathrm{eV}$ for lithium fluoride. The photon energy corresponding to the radiation of a femtosecond titanium-sapphire laser with $\lambda=800 \mathrm{~nm}$ is $\sim 1.5 \mathrm{eV}$. Therefore the formation of color centers in the crystals under study is due to multiphoton absorption of laser radiation energy by the electronic subsystem of matter with the formation of electron-hole pairs as a result of self-focusing and filamentation of the exciting femtosecond laser radiation [7]. The mechanism of the color centers creation under the influence of femtosecond laser radiation includes highly nonlinear generation of electron-hole pairs in the region of passage of the filaments, their recombination resulting with the formationif anionic excitons, decay of the excitons into the Frenkel defects by the Lushchik-Vitola-Hershey-Puli mechanism, their recharge, migration and aggregation.

It is shown that the axial dependence of energy accumulated by crystal under the influence of the single femtosecond pulses of laser radiation or the small series of them is "ragged", nonmonotonic in nature. The microscopic examination showed that the this is determined by the fact that the accumulation takes place in the areas of self-focusing filaments of laser radiation which are small in size (their length is $\sim 30 \mathrm{~mm}$, their diameter is $\sim 2 \mu \mathrm{m}$ ) in the initial stages of irradiation and their number is small. With increasing number of laser pulses the length and diameter of the spurs induced in the crystal by filaments also increase and the axial dependence of the accumulated energy is smoothed.

With increasing number of laser irradiation pulses the magnitude of the light sum of thermally stimulated luminescence emitted by irradiated crystals grows superlinear in the beginning then reaches a maximum and then decreases. The discovered effect of the accumulated light sum saturation is due to the fact that the concentration, aggregation degree and nomenclature of color centers are higher in the central paraxial part of the spurs than in the peripheral part.

The spectra of thermally stimulated luminescence of LiF crystals at different temperatures measured in the process of thermoluminescence show that the $\mathrm{F}_{2}$ centers are the main emission centers in the TSL process. One can assume that interstitial fluoride ions (I-centers) are the kinetic particles. They split off the $\mathrm{X}_{3}^{-}$centers in result of thermal decomposition of latter into the I-centers and molecules $\mathrm{X}_{2}{ }^{0}$. While recombining with $\mathrm{F}_{3}{ }^{+}$centers I-centers can form $\mathrm{F}_{2}$ centers in excited state, i.e., the excited centers of emission which provide the characteristic emission spectrum of $\mathrm{F}_{2}$ centers emitted in the form of thermally stimulated luminescence.

Work has been performed under the project of SB RAS II.10.1.6. «Mechanisms of extreme non-destructive interaction of solid dielectrics with intense laser radiation».

\section{REFERENCES}

[1] Martynovich, E.F., Glazunov, D.S., Grigorova, A.A., Starchenko, A.A., Kirpichnikov, A.V., Trunov, V.I., Merzlyakov, M.A., Petrov, V.V., Pestryakov, E.V., Opt.and Spec., V.105, 380 (2008).

[2] Martynovich, E.F., Balyunov, D.V., Kuznetsov, A.V., Kirpichnikov, A.V., Trunov, V.I., Pestryakov, E.V., Bagaev, S.N., Proc.of the universities, Physics, V. 52, N. 12/3. 198 (2009).

[3] Lushchyk, Ch.B. and Lushchyk, A.Ch., [The collapse of the electronic excitations with the formation of defects in solids], Science, Moscow, 263 (1989).

[4] Blunt, R.F., Cohen, M.I., Phys. Rev., V. 153, N 3, 1031 (1967).

[5] Sibley W.A., Facey O.E., Phys. Rev., V. 174, N 3, 1076 (1968).

[6] Baldacchini, G., Montereali, R.M,. Nichelatti, E,. Kalinov, V.S., Voitovich, A.P. et al., "Thermoluminescence in pure LiF crystals: Glow peaks and their connection with color centers," J. Appl. Phys, V. 104, (2008).

[7] Martynovich, E.F., Kuznetsov, A.V., Kirpichnikov, A.V., Pestryakov, E.V., Bagaev, S.N., Quantum Electronics, V. 43, N 5, 463 (2013). 